\title{
DETERMINANTAL FORMULAS FOR ORTHOGONAL AND SYMPLECTIC DEGENERACY LOCI
}

\author{
WILLIAM FULTON
}

\begin{abstract}
Given a vector bundle $V$ of rank $n$ on a variety $X$, together with two complete flags of subbundles, there is a degeneracy locus $X_{w} \subset X$ for each $w$ in the symmetric group $S_{n}$. With suitable genericity hypotheses, the class of $X_{w}$ in the Chow group of $X$ is given by a double Schubert polynomial in the first Chern classes of the quotient line bundles of the flags [9]. In this note we give similar formulas for corresponding loci when $V$ has an orthogonal or symplectic structure and the flags are isotropic; there is one such locus $X_{w}$ for each $w$ in the corresponding Weyl group.
\end{abstract}

\section{Introduction}

For any partition $\lambda=\left(\lambda_{1} \geq \lambda_{2} \geq \ldots \geq \lambda_{k}>0\right)$, and a formal sum $c_{0}+c_{1}+c_{2}+\ldots$ of commuting elements $c_{i}$ in a ring, with $c_{i}=0$ if $i<0$, denote by $\Delta_{\lambda}(c)$ the "Schur determinant"

$$
\Delta_{\lambda}(c)=\operatorname{det}\left(c_{\lambda_{i}+j-i}\right)_{1 \leq i, j \leq k} .
$$

The partition $(k, k-1, \ldots, 1)$ whose $i^{\text {th }}$ term is $k+1-i$ will be denoted by $\rho(k)$. In this introduction, for simplicity, we consider degeneracy loci on a nonsingular ambient variety $X$, with the assumption that the maps are generic enough so that all the loci have the expected codimension. We postpone to the next section the precise description of these loci as subschemes, and the statements without the assumptions of smoothness or genericity. In this introduction, formulas for these loci are given in the Chow rings with rational coefficients, but this can be improved to the Chow rings with integer coefficients.

Suppose $V$ is a vector bundle of rank $n$ on a nonsingular algebraic variety $X$, and $E$ and $F$ are subbundles of ranks $e$ and $f$.

For $k \leq \min (e, f)$, let $D_{k}$ be the locus in $X$ where the dimension of the intersection of the fibers $E(x) \cap F(x)$ is at least $k$. One form of the Giambelli-Thom-Porteous formula states that if $D_{k}$ has the expected

Received February 3, 1994 and, in revised form, October 31, 1994. 
codimension $d=k(n-e-f+k)$, then the class $\left[D_{k}\right]$ of $D_{k}$ in the Chow group $A^{d} X$ is given by the formula

$$
\left[D_{k}\right]=\Delta_{\lambda}(c),
$$

where $\lambda$ consists of the integer $n-e-f+k$ repeated $k$ times, and $c=c(V) / c(E) c(F)$.

Several years ago Joe Harris asked for an analogous formula for the case when $V$ is a bundle of rank $2 n$, equipped with a nondegenerate quadratic form, i.e., a symmetric bilinear map from $V \otimes V$ to a line bundle $L$ that is nondegenerate on each fiber. We call a bundle equipped with such a quadratic form an orthogonal bundle. Let $E$ and $F$ be subbundles of $V$ of rank $n$ that are (totally) isotropic, i.e., the quadratic form vanishes when restricted to each of them. Assume that $E$ and $F$ are in the same family, by which we mean that the dimension of the intersection $E(x) \cap F(x)$ is everywhere congruent to $n$ modulo 2. For $k \leq n$ and $k \equiv n(\bmod 2)$, one has a locus $D_{k}$ where the dimension of $E(x) \cap F(x)$ is at least $k$. If $D_{k}$ has the expected codimension $\left(\begin{array}{l}k \\ 2\end{array}\right)$, Harris asked for a formula for $\left[D_{k}\right]$. Our answer is the formula

$$
\left[D_{k}\right]=\Delta_{\rho(k-1)}(c), \quad c=\frac{1}{2}\left(c\left(E^{\vee} \otimes \sqrt{L}\right)+c\left(F^{\vee} \otimes \sqrt{L}\right)\right) .
$$

Here $E^{\vee}$ and $F^{\vee}$ denote the dual bundles, and $\sqrt{L}$ denotes a formal square root of the line bundle $L$; this square root enters the formula only through its first Chern class, which is $\frac{1}{2} c_{1}(L)$. The same formula is valid if $E$ and $F$ are in the opposite family, i.e., the dimension of $E(x) \cap F(x)$ is always congruent to $n-1$ modulo 2 , and $k$ is congruent to $n-1$ modulo 2 .

Similarly, if $V$ is an orthogonal bundle of rank $2 n+1$, and $E$ and $F$ are isotropic subbundles of rank $n$, then for any $k \leq n$, the locus $D_{k}$ where the dimension of intersection is at least $k$ is given by the formula

$$
\left[D_{k}\right]=\Delta_{\rho(k)}(c), \quad c=\frac{1}{2}\left(c\left(E^{\vee} \otimes \sqrt{L}\right)+c\left(F^{\vee} \otimes \sqrt{L}\right)\right) .
$$

We also prove a symplectic analogue. For this, $V$ is a symplectic vector bundle of rank $2 n$, i.e., $V$ is equipped with an everywhere nondegenerate skew-symmetric form $V \otimes V \rightarrow L$, and $E$ and $F$ are subbundles of rank $n$ that are Lagrangian, i.e., the restrictions of the skew form to $E$ and $F$ are identically zero. For $k \leq n$, one has the locus $D_{k}$ where the dimension of intersection is at least $k$, and in this case we have

$$
\left[D_{k}\right]=\Delta_{\rho(k)}(c), \quad c=c\left(E^{\vee} \otimes \sqrt{L}\right)+c\left(F^{\vee} \otimes \sqrt{L}\right) .
$$


The usual Giambelli-Thom-Porteous formula states that for a map $\varphi: E \rightarrow F$ of vector bundles, the locus $D_{r}(\varphi)$ where the rank of $\varphi$ is at most $r$ is given by the formula

$$
\left[D_{r}(\varphi)\right]=\Delta_{\lambda}(c), \quad c=c(F) / c(E)
$$

with $\lambda$ consisting of $e-r$ copies of $f-r$, where $e$ and $f$ are the ranks of $E$ and $F$. Formula (5) is equivalent to formula (1). Indeed, to deduce (1) from (5), consider the map from $E$ to $V / F$. Conversely, to deduce (5) from (1), given such a map $\varphi$, let $V=E \oplus F$, and consider the intersection of the graph $E_{\varphi}=\{e \oplus \varphi(e)\}$ of $\varphi$ with the bundle $E_{0}=E \oplus 0$.

Formulas (2) and (4) similarly imply known formulas for maps of vector bundles. If $E$ is a vector bundle of rank $n$ on $X, L$ a line bundle, and $\varphi: E \rightarrow E^{\vee} \otimes L$ is a skew-symmetric map of vector bundles, i.e., $\varphi^{\vee} \otimes i d_{L}:\left(E^{\vee} \otimes L\right)^{\vee} \otimes L=E \rightarrow E^{\vee} \otimes L$ is $-\varphi$, then the locus $D_{r}(\varphi)$ where the rank of $\varphi$ is at most $r$ is given by the formula

$$
\left[D_{r}(\varphi)\right]=\Delta_{\rho(n-r-1)}(c), \quad c=c\left(E^{\vee} \otimes \sqrt{L}\right)
$$

This is a result of Harris and Tu [14], with values in integral cohomology, and Jòzefiak, Lascoux, and Pragacz [15], when $L$ is trivial, and Pragacz [20] in general. To deduce this from (2), set $V=E \oplus\left(E^{\vee} \otimes L\right)$, with the symmetric pairing given by the formula $\left\langle e_{1} \oplus f_{1}, e_{2} \oplus f_{2}\right\rangle=f_{1}\left(e_{2}\right)+$ $f_{2}\left(e_{1}\right)$; in this formula we identify $E^{\vee} \otimes L$ with $\operatorname{Hom}(\mathrm{E}, \mathrm{L})$. The graph $E_{\varphi}$ of $\varphi$ and $E_{0}=E \oplus 0$ are isotropic subbundles, and $D_{r}(\varphi)$ is the locus $D_{n-r}$ given in (2). Note that here $F^{\vee}=E^{\vee}$, so

$$
\frac{1}{2}\left(c_{i}\left(E^{\vee} \otimes \sqrt{L}\right)+c_{i}\left(F^{\vee} \otimes \sqrt{L}\right)=c_{i}\left(E^{\vee} \otimes \sqrt{L}\right) .\right.
$$

Similarly, if $\varphi: E \rightarrow E^{\vee} \otimes L$ is a symmetric map, i.e., $\varphi^{\vee} \otimes i d_{L}=\varphi$, then the locus $D_{r}(\varphi)$ where the rank of $\varphi$ is at most $r$ is given by the formula

$$
\left[D_{r}(\varphi)\right]=2^{n-r} \cdot \Delta_{\rho(n-r)}(c), \quad c=c\left(E^{\vee} \otimes \sqrt{L}\right)
$$

To deduce this from (4), set $V=E \oplus\left(E^{\vee} \otimes \sqrt{L}\right)$, with the skewsymmetric pairing given by the formula $\left\langle e_{1} \oplus f_{1}, e_{2} \oplus f_{2}\right\rangle=f_{1}\left(e_{2}\right)-$ $f_{2}\left(e_{1}\right)$. This time the graph $E_{\varphi}$ of $\varphi$ and $E_{0}$ are Lagrangian subbundles, and $D_{r}(\varphi)$ is the locus $D_{n-r}$ given in (4). Note that these arguments are not reversible: formulas (2) and (4) do not follow from formulas (6) and (7).

In [9] a general formula for degeneracy loci was given that contains the Giambelli-Thom-Porteous formula as a special case. For a bundle $V$ of rank $n$ with two complete flags of subbundles, there is a degeneracy 
locus for each permutation in the symmetric group $S_{n}$, which is the Weyl group $W$ for type $\left(A_{n-1}\right)$. Our goal in this article is to produce analogous formulas for corresponding degeneracy loci for elements in the Weyl groups of the other classical types $\left(B_{n}\right),\left(C_{n}\right)$, and $\left(D_{n}\right)$. We want the formulas to be determinantal whenever possible, and the formulas should have similar shapes for all the classical groups.

Another motivation for this work is that, for type $\left(A_{n-1}\right)$, the answers to the general problem are the double Schubert polynomials invented by Lascoux and Schützenberger, whose combinatorial properties have been of considerable interest (cf. [17], [4]). There is interest and recent progress in finding such polynomials for the other groups $S p_{2 n}$ and $S O_{m}$ (see [3] for the case of single Schubert polynomials).

We turn now to the general statements of our results. In each case one is given a vector bundle $V$ of rank $m$ on a variety $X$, together with flags of subbundles

$$
\begin{aligned}
& E_{\bullet}: 0=E_{0} \subset E_{1} \subset E_{2} \subset \ldots \subset E_{n}=E \subset V \\
& F_{\bullet}: 0=F_{0} \subset F_{1} \subset F_{2} \subset \ldots \subset F_{n}=F \subset V .
\end{aligned}
$$

The situations considered are:
$\left(A_{n-1}\right) \quad m=n$,
no conditions on $V$ or the flags;
$\left(B_{n}\right) \quad m=2 n+1, \quad V$ is orthogonal, $E$ and $F$ are isotropic;
$\left(C_{n}\right) \quad m=2 n, \quad V$ is symplectic, $E$ and $F$ are Lagrangian;
$\left(D_{n}\right) \quad m=2 n$,
$V$ is orthogonal, $E$ and $F$ are isotropic, and $\operatorname{dim}(E(x) \cap F(x) \equiv n(\bmod 2)$
for all $x$ in $X$.

In each case the flags can be extended to complete flags. In case $\left(B_{n}\right)$, set $E_{n+i}=E_{n+1-i}^{\perp}$ and $F_{n+i}=F_{n+1-i}^{\perp}$ for $i \geq 1$; in cases $\left(C_{n}\right)$ and $\left(D_{n}\right)$, set $E_{n+i}=E_{n-i}^{\perp}$ and $F_{n+i}=F_{n-i}^{\perp}$ for $i \geq 1$. We will use the following realizations of the Weyl groups $W$ :

$$
\begin{aligned}
& \left(A_{n-1}\right) \quad W=S_{n} \\
& \left(B_{n}\right) \quad W=\left\{w \in S_{2 n+1}: w(i)+w(2 n+2-i)=2 n+2 \text { for all } i\right\} \\
& \left(C_{n}\right) \quad W=\left\{w \in S_{n}: w(i)+w(2 n+1-i)=2 n+1 \text { for all } i\right\} \\
& \left(D_{n}\right) \quad W=\left\{w \in S_{2 n}: w(i)+w(2 n+1-i)=2 n+1 \text { for all } i\right. \text {, and } \\
& \text { the number of } i \leq n \text { such that } w(i)>n \text { is even }\} \text {. }
\end{aligned}
$$

Each Weyl group $W$ is generated by a standard set of involutions. In each case one has transpositions $s_{i}$ that interchange $i$ and $i+1$ : in case $\left(A_{n-1}\right), s_{i}=(i, i+1)$ interchanges only $i$ and $i+1$; in case $\left(B_{n}\right) s_{i}$ also 
interchanges $2 n+1-i$ and $2 n+2-i$, while in cases $\left(C_{n}\right)$ and $\left(D_{n}\right) s_{i}$ must also interchange $2 n-i$ and $2 n+1-i$. The generators are

$$
\begin{aligned}
& \left(A_{n-1}\right) s_{1}, \ldots, s_{n-1} \\
& \left(B_{n}\right) \quad s_{1}, \ldots, s_{n-1} \text {, and } s_{n}=(n, n+2) \text {; } \\
& \left(C_{n}\right) \quad s_{1}, \ldots, s_{n-1}, \text { and } s_{n}=(n, n+1) \text {; } \\
& \left(D_{n}\right) \quad s_{1}, \ldots, s_{n-1} \text {, and } s_{n}=(n-1, n+1)(n, n+2) \text {. }
\end{aligned}
$$

The length $\ell(w)$ of $w$ in $W$ is the minimal number $\ell$ such that $w$ can be written as a product of $\ell$ of these generators. With this notation, the lengths have simple descriptions; in each of the following sets, the integers $i$ and $j$ are taken from the set $\{1, \ldots, n\}$ :

$$
\begin{array}{ll}
\left(A_{n-1}\right) & \ell(w)=\#\{i<j: w(i)>w(j)\} ; \\
\left(B_{n}\right) & \ell(w)=\#\{i<j: w(i)>w(j)\}+\#\{i \leq j: w(i)+w(j)>2 n+2\} ; \\
\left(C_{n}\right) & \ell(w)=\#\{i<j: w(i)>w(j)\}+\#\{i \leq j: w(i)+w(j)>2 n+1\} ; \\
\left(D_{n}\right) & \ell(w)=\#\{i<j: w(i)>w(j)\}+\#\{i<j: w(i)+w(j)>2 n+1\} .
\end{array}
$$

If the inequality in each of these conditions is reversed, one gets a number we call the codimension of $w$ and denote by $\operatorname{cod}(w)$; for example, in case $\left(B_{n}\right)$,

$$
\operatorname{cod}(w)=\#\{i<j: w(i)<w(j)\}+\#\{i \leq j: w(i)+w(j)<2 n+2\} .
$$

The sum of $\ell(w)$ and $\operatorname{cod}(w)$ is the maximal length of an element of $W$, which is the dimension of the corresponding flag manifold. These dimensions are: $n(n-1) / 2$ for $\left(A_{n-1}\right), n^{2}$ for $\left(B_{n}\right)$ or $\left(C_{n}\right)$, and $n^{2}-n$ for $\left(D_{n}\right)$.

There are operators $\partial_{i}$ defined on polynomials in variables $x_{1}, \ldots, x_{n}$, with coefficients in any commutative ring, coming from [1] and [6]. For $1 \leq i \leq n-1$, these operators are defined by

$$
\partial_{i}(P)=\left(P\left(x_{1}, \ldots, x_{n}\right)-P\left(x_{1}, \ldots x_{i-1}, x_{i+1}, x_{i}, x_{i+2}, \ldots x_{n}\right)\right) /\left(x_{i}-x_{i-1}\right) .
$$

For our use, the other operators $\partial_{n}$ involve another variable $z$. These are defined by:

$$
\begin{aligned}
\left(B_{n}\right) \partial_{n}(P)= & 2\left(P\left(x_{1}, \ldots, x_{n}\right)-P\left(x_{1}, \ldots, x_{n-1},-x_{n}-z\right)\right) /\left(2 x_{n}+z\right) ; \\
\left(C_{n}\right) \partial_{n}(P)= & \left(P\left(x_{1}, \ldots, x_{n}\right)-P\left(x_{1}, \ldots, x_{n-1},-x_{n}-z\right)\right) /\left(2 x_{n}+z\right) ; \\
\left(D_{n}\right) \partial_{n}(P)= & \left(P\left(x_{1}, \ldots, x_{n}\right)\right. \\
& \left.-P\left(x_{1}, \ldots, x_{n-2},-x_{n}-z,-x_{n-1}-z\right)\right) /\left(x_{n-1}+x_{n}+z\right) .
\end{aligned}
$$

For any $w$ in $W$, define an operator $\partial_{w}$ by the formula

$$
\partial_{w}=\partial_{i_{1}} \circ \ldots \circ \partial_{i_{\ell}} \quad \text { if } \quad w=s_{i_{1}} \cdot \ldots \cdot s_{i_{\ell}}, \quad \ell=\ell(w) \text {. }
$$


The operators $\partial_{i}$ satisfy relations corresponding to the Coxeter relations defined by the generators $s_{i}$, which implies that the definition of $\partial_{w}$ is independent of the choice.

With these conventions, we can give a uniform description of a degeneracy locus $X_{w} \subset X$ for each $w \in W$. As a first approximation, $X_{w}$ can be described as the locus of $x \in X$ such that

$$
\operatorname{dim}\left(E_{p}(x) \cap F_{q}(x)\right) \geq \#\{i \leq p: w(i) \leq q\} \quad \text { for all } \quad 1 \leq p, q \leq m ;
$$

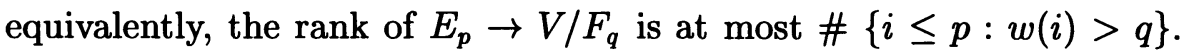
In all cases, if the flags are sufficiently generic, the precise definition of $X_{w}$ is as the closure of the set $X_{w}^{o}$ on which the dimension of $E_{p} \cap F_{q}$ is equal to \# $\{i \leq p: w(i) \leq q\}$ for all $p$ and $q$, with its reduced structure. With these assumptions, $X_{w}$ has pure codimension $\operatorname{cod}(w)$. The precise definition of $X_{w}$ as a subscheme of $X$, without any assumptions of smoothness or genericity, will be given in the next section.

Our formulas for these loci involve certain Chern classes. Set

$$
x_{i}=-c_{1}\left(E_{i} / E_{i-1}\right), \quad y_{i}=-c_{1}\left(F_{n+1-i} / F_{n-1}\right), \quad 1 \leq i \leq n,
$$

which are classes in the Chow group $A^{1} X$. Let $z=c_{1}(L)$, and let $v=\frac{1}{2} z=c_{1}(\sqrt{L})$. Define classes $c_{i}$ in $A^{i} X$ by the formulas

$$
\begin{aligned}
& \left(B_{n}\right) \quad c_{i}=\frac{1}{2}\left(e_{i}\left(x_{1}+v, \ldots, x_{n}+v\right)+e_{i}\left(y_{1}+v, \ldots, y_{n}+v\right)\right), \quad 0 \leq i \leq n ; \\
& \left(C_{n}\right) \quad c_{i}=e_{i}\left(x_{1}+v, \ldots, x_{n}+v\right)+e_{i}\left(y_{1}+v, \ldots, y_{n}+v\right), \quad 0 \leq i \leq n ; \\
& \left(D_{n}\right) \quad c_{i}=\frac{1}{2}\left(e_{i}\left(x_{1}+v, \ldots, x_{n}+v\right)+e_{i}\left(y_{1}+v, \ldots, y_{n}+v\right)\right), \quad 0 \leq i \leq n-1 .
\end{aligned}
$$

In each of these formulas, $e_{i}\left(z_{1}, \ldots, z_{n}\right)$ denotes the $i^{\text {th }}$ elementary symmetric polynomial in variables $z_{1}, \ldots, z_{n}$.

Define a polynomial $\Delta$ in these variables by the formulas: $\Delta=1$ in case $\left(A_{n-1}\right) ; \Delta=\Delta_{\rho(n)}(c)$ in case $\left(B_{n}\right)$ or $\left(C_{n}\right)$; and $\Delta=\Delta_{\rho(n-1)}(c)$ in case $\left(D_{n}\right)$. In each case the variables $c_{i}$ are as prescribed above, with $c_{i}$ defined to be zero for $i$ not specified. Define polynomials $P_{w}$, for $w$ in $W$, by the following rule: write $w=s_{i_{1}} \cdot \ldots \cdot s_{i_{\ell}}$ with $\ell=\ell(w)$, and set

$$
P_{w}=\partial_{i_{1}} \circ \ldots \circ \partial_{i_{\ell}}\left(\prod_{i+j \leq n}\left(x_{i}-y_{j}\right) \cdot \Delta\right) .
$$

Equivalently, $P_{w}=\partial_{w^{-1}}\left(\prod_{i+j \leq n}\left(x_{i}-y_{j}\right) \cdot \Delta\right)$. With the above interpretation of the variables, $P_{w}$ defines a class in $A^{\operatorname{cod}(w)}(X)$.

Theorem. The class of $X_{w}$ in $A^{\operatorname{cod}(w)}(X)$ is given by the formula

$$
\left[X_{w}\right]=P_{w} \text {. }
$$


Formulas (1)-(4) can be regarded as special cases of this theorem, although at present they are proved together with the theorem. The theorem can be applied in the same way to obtain formulas for the degrees of varieties in projective space defined by symmetric or skewsymmetric matrices of general forms satisfying various rank conditions, as begun by Giambelli [13]. The pattern for this is similar to that worked out for general matrices in [9]. To carry this out, each $E_{i}$ is a direct sum of line bundles on projective space, and one is intersecting $E$ with the graph of the map from $E$ to $E^{\vee} \otimes L$ determined by the matrix of forms. In this case, however, it only happens that certain of the degeneracy loci are irreducible, or even of the right dimension.

There are analogous formulas for the case where $V$ is orthogonal of rank $2 n$, and $E$ and $F$ are isotropic with $\operatorname{dim}(E(x) \cap F(x)) \equiv n-1$ $(\bmod 2)$, a situation we denote by $\left(\widetilde{D}_{n}\right)$. In the presense of suitable positivity assumptions on the bundles, one can deduce from our formulas that some degeneracy loci must be nonempty.

The theorem, both for its precise statement and its proof, is obtained by pulling back the corresponding formula for Schubert varieties in flag bundles, which is proved in [10]. This reduction is carried out in the next section. This includes a description of the loci $X_{w}$ in general, and how the theorem extends to arbitrary varieties, and with values in the Chow rings with integer coefficients. A last section describes some applications.

Both the discovery of the formula and the proof of the theorem depend on the work of D. Edidin and W. Graham [7], whom we thank for this work and for many useful discussions. We also thank J. Harris for stimulating this work, and A. Bertram, R. Kottwitz, R. MacPherson and R. Narasimhan for useful suggestions. This research was supported in part by NSF Grants DMS 9007575 and 9307922.

\section{Flag bundles}

The definition of the locus $X_{w}$ and the proof of the theorem will be obtained by pulling them back from the "universal" case, which takes place on a flag bundle. For this, we modify our assumptions. Now $X$ is an arbitrary irreducible variety, $V$ is a vector bundle on $X$, with a nondegenerate bilinear skew-symmetric or symmetric form $V \otimes V \rightarrow L$ in cases $\left(C_{n}\right),\left(B_{n}\right)$, or $\left(D_{n}\right)$, and we have a flag $F$. of subbundles of $V$ on $X$ :

$$
0=F_{0} \subset F_{1} \subset F_{2} \subset \ldots \subset F_{n}=F \subset V .
$$

We assume that $V$ and this flag satisfy the conditions of the introduc- 
tion, depending on the type $\left(A_{n-1}\right),\left(B_{n}\right),\left(C_{n}\right)$, or $\left(D_{n}\right)^{1}$ Let $\mathcal{F}=\mathrm{Fl}(V)$ be the corresponding flag bundle of such flags. This comes equipped with a projection $\rho: \mathcal{F} \rightarrow X$, and a universal flag $U$. of subbundles

$$
0=U_{0} \subset U_{1} \subset U_{2} \subset \ldots \subset U_{n}=U \subset \rho^{*}(V),
$$

with $U$ Lagrangian in case $\left(C_{n}\right)$ and isotropic in cases $\left(B_{n}\right)$ and $\left(D_{n}\right)$. In case $\left(D_{n}\right)$, in addition, we require that the dimension of $U(y) \cap F(\rho(y))$ be congruent to $n$ modulo 2 for all $y$ in $\mathcal{F}$; this added condition makes the flag bundle connected. The fiber of $\mathcal{F}$ over a point in $X$ is the usual flag manifold for the corresponding classical group.

We next describe the Schubert varieties $\mathfrak{X}_{w} \subset \mathcal{F}$ for each $w \in W$. In each case, $\mathfrak{X}_{w}$ is the closure of the set $\mathfrak{X}_{w}^{o}$ of points $y$ in $\mathcal{F}$ on which the dimension of $U_{p}(y) \cap F_{q}(\rho(y))$ is equal to the cardinality of the set $\{i \leq p: w(i) \leq q\}$ for all $p$ and $q$, with its reduced structure. Each $\mathfrak{X}_{w}$ is an irreducible variety, of codimension $\operatorname{cod}(w)$, in $\mathcal{F}$. Note that $\mathcal{F}$ is a locally trivial bundle over $X$, and $\mathfrak{X}_{w}$ is locally the product of an open set in $X$ with a classical Schubert variety. For equations for these Schubert varieties, see [16] and its references.

Let $u_{i}=-c_{1}\left(U_{i} / U_{i-1}\right)$ for $1 \leq i \leq n, y_{i}=-c_{i}\left(F_{n+1-i} / F_{n-i}\right), v=$ $\frac{1}{2} c_{1}(L)$. The universal case of the theorem of the introduction is the following:

Theorem 1. The class of $\mathfrak{X}_{w}$ in $A^{\operatorname{cod}(w)}(\mathcal{F})$ is given by the formula

$$
\left[\mathfrak{X}_{w}\right]=P_{w}\left(u_{1}+v, \ldots, u_{n}+v, y_{1}+v, \ldots, y_{n}+v\right)
$$

To apply the theorem in the general context, suppose we are given, in addition to $V$ with its flag $F_{\text {. on }} X$, another flag $E_{\text {. of subbun- }}$ dles satisfying the same conditions as $F_{\bullet}$, with the added condition in case $\left(D_{n}\right)$ that $E_{n}$ and $F_{n}$ are in the same family. The flag $E_{\bullet}$ corresponds to a section $s: X \rightarrow \mathcal{F}$ of $\mathcal{F} \rightarrow X$ such that $s^{*}\left(U_{\bullet}\right)=E_{\bullet}$. The degeneracy locus $X_{w}$ in $X$ can then be given a precise definition and scheme-theoretic structure by defining $X_{w}$ to be the inverse image $s^{-1}\left(\mathfrak{X}_{w}\right)$ of the universal Schubert variety $\mathfrak{X}_{w} \subset \mathcal{F}$.

We can define the class $\mathbb{X}_{w}$ of this locus to be the intersection class $s^{!}\left[\mathfrak{X}_{w}\right]$, which is a class in the Chow group $A_{m}\left(X_{w}\right)$, where $m=\operatorname{dim}(X)-$ $\operatorname{cod}(w)$; this class is the refined intersection class constructed by the procedure of $[8, \S 6.2]$, which is defined because $s$ is a regular embedding.

${ }^{1}$ If the ground field has characteristic 2, the definitions must be modified as usual in cases $\left(B_{n}\right)$ and $\left(D_{n}\right)$. We are given a quadratic map $N: V \rightarrow L$, with $N(\lambda v)=\lambda^{2} N(v)$ for sections $v$ and functions $\lambda$, such that the map $Q(u, v)=N(u+v)-N(u)-N(v)$ is bilinear. The restriction of $N$ to the bundle $F=F_{n}$ must vanish identically. 
Corollary 1. The image of $\mathbb{X}_{w}$ in $A_{m}(X)$ is $P_{w} \cap[X]$, where $P_{w}$ is the polynomial

$$
P_{w}=\partial_{w^{-1}}\left(\prod_{i+j \leq n}\left(x_{i}-y_{j}\right) \cdot \Delta\right) .
$$

If $\operatorname{codim}\left(X_{w}, X\right)=\operatorname{cod}(w)$, and $X$ is Cohen-Macaulay, then $X_{\omega}$ is the class of the subscheme $X_{w}$, i.e., $\mathbb{X}_{w}=\left[X_{\omega}\right]$.

It follows, for example, that $X_{w}$ is nonempty whenever the class $P_{w} \cap[X]$ is nonzero.

There are similar formulas for an orthogonal bundle of rank $2 n$ when $E=E_{n}$ and $F=F_{n}$ are in the opposite family, a case that may be denoted by $\left(\widetilde{D}_{n}\right)$. For this, set

$$
\begin{aligned}
\widetilde{W}= & \left\{\widetilde{w} \in S_{2 n}: \widetilde{w}(i)+\widetilde{w}(2 n+1-i)=2 n+1 \text { for all } i,\right. \\
& \text { and the number of } i \leq n \text { such that } \widetilde{w}(i)>n \text { is odd }\} .
\end{aligned}
$$

For each $\widetilde{w}$ in $\widetilde{W}$ there is a locus $X_{\widetilde{w}}$ defined by the same conditions as in the other cases: the dimension of $E_{p}(x) \cap F_{q}(x)$ is at least the cardinality of $\{i \leq p: \widetilde{w}(i) \leq q\}$ for all $1 \leq p, q \leq 2 n$. Although $\widetilde{W}$ is not a subgroup of $S_{2 n}$, the same formula as for $\left(D_{n}\right) \operatorname{defines} \operatorname{cod}(\widetilde{w})$ for $\widetilde{w}$ in $\widetilde{W}$. There is a one-to-one correspondence between the Weyl group $W$ for $\left(D_{n}\right)$ and $\widetilde{W}$ : given $w \in W$, define $\widetilde{w}$ by the formula $\widetilde{w}(i)=w(i)$ for $i \notin\{n, n+1\}$, and $\widetilde{w}(i)=2 n+1-w(i)$ for $i \in\{n, n+1\}$. This correspondence preserves codimension.

Given an isotropic flag $E_{\bullet}$, there is a unique isotropic flag $\widetilde{E}_{\bullet}$ defined by setting $\widetilde{E}_{i}=E_{i}$ for $i \leq n-1$, defining $\widetilde{E}_{n}$ so that $E_{n-1}^{\perp} / E_{n-1}$ is the direct sum of $E_{n} / E_{n-1}$ and $\widetilde{E}_{n} / E_{n-1}$. If $E_{n}$ and $F$ are in the opposite family, then $\widetilde{E}_{n}$ and $F$ are in the same family, and $E_{\bullet}$ is in $X_{\widetilde{w}}$ exactly when $\widetilde{E}_{\bullet}$ is in $X_{w}$. We can therefore give $X_{\widetilde{w}}$ its precise definition and scheme-theoretic structure by letting $s: X \rightarrow \mathcal{F}$ be the section of the flag bundle $\mathcal{F}$ for $\left(D_{n}\right)$ such that $s^{*}\left(U_{\bullet}\right)=\widetilde{E}_{\bullet}$, and defining $X_{\widetilde{w}}$ to be $s^{-1}\left(\mathfrak{X}_{w}\right)$. Similarly, we have the class $\mathbb{X}_{\tilde{w}}=s^{!}\left[\mathfrak{X}_{w}\right]$. For $w$ in $W$, define $P_{\widetilde{w}}$ by the formula

$$
P_{\widetilde{w}}\left(x_{1}, \ldots, x_{n}, y_{1}, \ldots, y_{n}\right)=P_{w}\left(x_{1}, \ldots,-x_{n}-z, y_{1}, \ldots, y_{n}\right) .
$$

With the same definition of $x_{i}, y_{i}$, and $z$ as before, we obtain the following variation of Corollary 1 .

Corollary 2. The image of $\mathbb{X}_{\tilde{w}}$ in $A_{m}(X)$ is $P_{\widetilde{w}} \cap[X]$. If $\operatorname{cod}\left(X_{\widetilde{w}}, X\right)$ $=\operatorname{cod}(\widetilde{w})$, and $X$ is Cohen-Macaulay, then $\mathbb{X}_{\tilde{w}}=\left[X_{\tilde{w}}\right]$.

Similarly, the loci $D_{k}$ are the inverse images by the section $s$ of universal loci $\mathcal{D}_{k}=\left\{y \in \mathcal{F}: \operatorname{dim}(U(y) \cap F(\rho(y)) \geq k\}\right.$; in case $\left(D_{n}\right)$ we assume $k \equiv n(\bmod 2)$, and in case $\left(\widetilde{D_{n}}\right)$ we assume $k \equiv n-1(\bmod 2)$. 
Each $\mathcal{D}_{k}$ is one of the Schubert varieties in $\mathcal{F}$. As such, its class is given determined by Theorem 1, but we have the following explicit formulas:

Theorem 2. The class of $\mathcal{D}_{k}$ is given by the formula

$$
\begin{array}{llll}
\left(B_{n}\right) & {\left[\mathcal{D}_{k}\right]=\Delta_{\rho(k)}(c),} & & c=\frac{1}{2}\left(c\left(U^{\vee} \otimes \sqrt{L}\right)+c\left(F^{\vee} \otimes \sqrt{L}\right)\right) ; \\
\left(C_{n}\right) & {\left[\mathcal{D}_{k}\right]=\Delta_{\rho(k)}(c),} & & c=c\left(U^{\vee} \otimes \sqrt{L}\right)+c\left(F^{\vee} \otimes \sqrt{L}\right) \\
\left(D_{n}\right) & {\left[\mathcal{D}_{k}\right]=\Delta_{\rho(k-1)}(c),} & c=\frac{1}{2}\left(c\left(U^{\vee} \otimes \sqrt{L}\right)+c\left(F^{\vee} \otimes \sqrt{L}\right)\right) .
\end{array}
$$

As before, the schemes $D_{k} \subset X$ can be defined to be $s^{-1}\left(\mathcal{D}_{k}\right)$, and we can define cycle classes $\mathbb{D}_{k}$ in $A_{*}\left(D_{k}\right)$ to be $s^{\prime}\left[\mathcal{D}_{k}\right]$.

Corollary. The image of $\mathbb{D}_{k}$ in $A_{*}(X)$ is:

$$
\begin{array}{lll}
\left(B_{n}\right) & \Delta_{\rho(k)}(c) \cap[X], & c=\frac{1}{2}\left(c\left(E^{\vee} \otimes \sqrt{L}\right)+c\left(F^{\vee} \otimes \sqrt{L}\right)\right) . \\
\left(C_{n}\right) & \Delta_{\rho(k)}(c) \cap[X], & c=c\left(E^{\vee} \otimes \sqrt{L}\right)+c\left(F^{\vee} \otimes \sqrt{L}\right) \\
\left(D_{n}\right) \text { or }\left(\widetilde{D}_{n}\right) & \Delta_{\rho(k-1)}(c) \cap[X], & c=\frac{1}{2}\left(c\left(E^{\vee} \otimes \sqrt{L}\right)+c\left(F^{\vee} \otimes \sqrt{L}\right)\right)
\end{array}
$$

If the codimension of $D_{k}$ in $X$ is $\left(\begin{array}{c}k+1 \\ 2\end{array}\right)$ in cases $\left(B_{n}\right)$ or $\left(C_{n}\right)$, or $\left(\begin{array}{l}k \\ 2\end{array}\right)$ in case $\left(D_{n}\right)$ or $\left(\widetilde{D}_{n}\right)$, and $X$ is Cohen-Macaulay, then $\mathbb{D}_{k}=\left[D_{k}\right]$

As in [9], the proof of Theorem 1 has two main steps. One step is to show that $\left[\mathfrak{X}_{w^{\prime}}\right]=\partial_{i}\left(\left[\mathfrak{X}_{w}\right]\right)$ if $w^{\prime}=w \cdot s_{i}$ and $\ell\left(w^{\prime}\right)=\ell(w)+1$. The proof of this is almost the same as that in [9]. The other step is to prove the formula for the case where $w$ is the identity element in $W$, so $\mathfrak{X}_{w}$ is the locus where $U_{\bullet}=\rho^{*}\left(F_{\bullet}\right)$. To prove this, it suffices to prove that the locus on $\mathcal{F}$ where $U$ is equal to $\rho^{*}(F)$ is given by the polynomial $\Delta$, for then, on this locus, by the $\left(A_{n-1}\right)$ case and the projection formula, the locus where $U_{\bullet}=\rho^{*}\left(F_{\bullet}\right)$ is given by the product of $\Delta$ with $\prod_{i+j \leq n}\left(u_{i}-y_{j}\right)$. In the case $\left(A_{n-1}\right)$ there is a vector bundle of rank equal to the relative dimension of $\mathcal{F}$ over $X$, together with a section of this bundle whose zero locus is exactly $\mathfrak{X}_{i d}$, and whose top Chern class is $\prod_{i+j \leq n}\left(u_{i}-y_{j}\right)$. We know of no such bundle and section in the other cases. In these cases the formula can be proved by finding another expression for this locus, and doing some algebra to prove that this expression agrees with $\Delta$. (Note, however, that these equations are not equations of polynomials; they are valid only in the Chow rings of the flag bundles.) The same ideas are used to prove Theorem 2. By describing the Chow rings of the flag bundles with integer coefficients, one sees also that the polynomials $P_{w}$ have canonical expressions in the Chow rings with integer coefficients. For details, see [10].

When we informed P. Pragacz of the determinantal formula of the theorem, he reported that he and J. Ratajski [24] have another formula for the locus $\mathcal{D}_{n}$ where $U=\rho^{*} F$. They deduce this from a formula for the class of the diagonal in $\mathcal{G} \times{ }_{X} \mathcal{G}$ as a sum of products of Schur $Q$-polynomials ( $P$-polynomials in cases $\left(B_{n}\right)$ and $\left.\left(D_{n}\right)\right)$, where $\mathcal{G}$ is the 
corresponding Grassmann bundle. Again, some algebra will be required to show that this class is equal to the class $\Delta$ in the Chow rings, and to deduce our formulas for the other loci $\mathcal{D}_{k}$. In neither their work nor ours, however, do the determinants arise naturally, nor is it clear without some algebra why the answer should be expressed as any polynomial in the sums of Chern classes of the bundles.

For the case $\left(A_{n-1}\right)$, the algebra of double Schubert polynomials has been sufficiently developed so that one has simple determinantal formulas for a large number of the loci $\mathfrak{X}_{w}$ or $X_{w}$ (cf. [17] and [9]). This algebra has not yet been done for the cases $\left(B_{n}\right),\left(C_{n}\right)$, and $\left(D_{n}\right)$.

The theory of single Schubert polynomials for the other classical groups is currently being developed, particularly by Billey and Haiman [3]. As in the case $\left(A_{n-1}\right)$, there are well defined polynomials in certain variables, with a stability as $\mathrm{n}$ increases. We hope that this will extend to double polynomials; it would be interesting to compare the resulting polynomials with the classes $P_{w}$ defined here. ${ }^{2}$

\section{Applications}

The theorem of [12] can be used to show that, in some cases, some of these polynomials are positive, so the corresponding loci are nonempty. For example, if $E$ and $F$ have equal Chern classes, then $c=$ $2 c\left(E^{\vee} \otimes \sqrt{L}\right)$ in case $\left(C_{n}\right)$, and $c=2 c\left(E^{\vee} \otimes \sqrt{L}\right)$ in cases $\left(B_{n}\right)$ or $\left(D_{n}\right)$, so the determinants are, up to multiplying by a power of 2 , the Schur determinants of the bundle $E^{\vee} \otimes \sqrt{L}$ Hence we have:

Proposition. Suppose $c\left(E^{\vee} \otimes \sqrt{L}\right)=c\left(F^{\vee} \otimes \sqrt{L}\right)=c(G)$, where $G$ is an ample vector bundle on $X$, and $\left(\begin{array}{c}k+1 \\ 2\end{array}\right) \leq \operatorname{dim}(X)$ in case $\left(B_{n}\right)$ or $\left(C_{n}\right)$ or $\left(\begin{array}{l}k \\ 2\end{array}\right) \leq \operatorname{dim}(X)$ in case $\left(D_{n}\right)$ or $\left(\widetilde{D}_{n}\right)$. Then $D_{k}$ is nonempty.

For example, if $\varphi: E \rightarrow E^{\vee}$ is a symmetric or skew-symmetric map of vector bundles, with $E^{\vee}$ ample, one recovers the fact that the degeneracy loci $D_{r}(\varphi)$ must be nonempty whenever their expected dimensions are nonnegative. In applying this one need not take values in the Chow rings; they are stronger if values are taken in cohomology with rational coefficients, or in cycles modulo numerical equivalence.

Following a suggestion of A. Bertram, our formula can also be used to give another proof of his result on the nonemptiness of the loci $V^{r}$ of special divisors on a Prym variety [2]. This has also been carried out by

\footnotetext{
${ }^{2}$ Note that even in the $\left(A_{n-1}\right)$ case, the $P_{w}$ are not the usual double Schubert polynomials, due to the change of variables $x_{i}$ for $-x_{n+1-i}$; and $y_{i}$ for $-y_{n+1-i}$, as well as the change from $w$ to $w \cdot w_{o}$. These changes were made to obtain unified formulas for all the classical groups.
} 
De Concini and Pragacz [5], based on the results of [24]. We conclude this article by showing how to recover the formula of [5] for the class of $V^{r}$ from our determinantal formula.

We recall Mumford's realization of $V^{r}$ as a degeneracy locus, cf. [5]. One is given a two-sheeted étale covering $C^{\prime} \rightarrow C$ of a curve $C$ of genus $g$. The Prym variety $P \subset \mathrm{Jac}^{2 g-2}\left(C^{\prime}\right)$ is the locus of line bundles whose norm is $\omega_{C} ; P$ is a disjoint union of varieties $P^{+}$and $P^{-}$of dimension $g-1$, and $V^{r}$ is the subvariety of line bundles $L$ (in $P^{+}$if $\mathrm{r}$ is odd and in $P^{-}$if $r$ is even) on which $h^{0}\left(C^{\prime}, L\right)$ is at least $r+1$. Let $\mathcal{L}$ be a Poincaré bundle on $P \times C^{\prime}$ ), and let $D$ be a divisor on $C^{\prime}$. Set

$$
\begin{aligned}
& V=p_{*}\left(\mathcal{L} \otimes q^{*}(\mathcal{O}(D)) / \mathcal{L} \otimes q^{*}(\mathcal{O}(-D))\right. \\
& E=p_{*}\left(\mathcal{L} \otimes q^{*}(\mathcal{O}(D))\right), \quad F=p_{*}\left(\mathcal{L} / \mathcal{L} \otimes q^{*}(\mathcal{O}(-D))\right.
\end{aligned}
$$

For a suitable normalization of $\mathcal{L}$, and suitable choice of $D$, one has a symmetric non-degenerate pairing $V \otimes V \rightarrow \mathbb{1}, V$ is a vector bundle of rank $2 n$, and $E$ and $F$ are isotropic subbundles of rank $n$, where $n$ is the degree of $D$. The locus $V^{r}$ of special divisors is the locus where $E$ and $F$ meet in dimension at least $r+1$. By the theorem, the (virtual) formula for this locus is $2^{-r} \Delta_{\rho(r)}(c)$, where $c=c\left(E^{\vee}\right)+c\left(F^{\vee}\right)$.

Now $c_{i}\left(F^{\vee}\right)=0$ for $i>0$, and $E^{\vee}$ is ample [11]. The polynomial $\Delta_{\rho(r)}(c)$ need not be a positive polynomial in the Chern classes of $E^{\vee}$, however, so the theorem of [12] does not apply ${ }^{3}$. One can calculate the polynomial $\Delta_{\rho(r)}(c)$ in terms of the restriction $\Theta$ of the class of the theta divisor on $\mathrm{Pic}^{2 g-2}\left(C^{\prime}\right)$ to $J$, since one knows that $c_{i}\left(E^{\vee}\right)=(1 / i !) \Theta^{i}$. The result of De Concini and Pragacz is:

Proposition. The virtual class of $V^{r}$ is $d \cdot \Theta^{N}$, where $N=\left(\begin{array}{c}r+1 \\ 2\end{array}\right)$, and

$$
d=2^{-r} \prod_{i=1}^{r} \frac{(i-1) !}{(2 i-1) !}
$$

Since $\Theta$ is an ample divisor, and $d \neq 0$, this implies that $V^{r}$ is nonempty whenever $\left(\begin{array}{c}r+1 \\ 2\end{array}\right) \leq g-1$.

Proof. By the preceding remarks, it suffices to prove that $\Delta_{\rho(r)}(c)=$ $\prod_{i=1}^{r}(i-1) ! /(2 i-1) !$, where $c_{i}$ is defined to be $1 / i$ ! if $i>0, c_{0}=2$, and $c_{i}=0$ for $i<0$. Start with the $r \times r$ matrix whose determinant is defined to be $\Delta_{\rho(r)}(c)$. Reverse the numberings in the rows and columns, and then multiply the $i^{\text {th }}$ row by $(2 i-1)$ ! and divide the $j^{\text {th }}$ column by

${ }^{3}$ For example, $\Delta_{\rho(2)}(c)=P_{(2,1)}\left(c\left(E^{\vee}\right)\right)-P_{(3)}\left(c\left(E^{\vee}\right)\right)$, where $P_{\lambda}$ denotes the (positive) Schur polynomial corresponding to the partition $\lambda$. 
$(j-1)$ !. The resulting matrix $C$ has entries $c_{i j}$ defined by the formula

$$
c_{i j}=\left\{\begin{array}{cl}
\left(\begin{array}{c}
2 i-1 \\
2 i-j
\end{array}\right) & \text { if } j<2 i \\
2 & \text { if } j=2 i \\
0 & \text { if } j>2 i .
\end{array}\right.
$$

To prove the proposition it suffices to show that $\operatorname{det}(C)=1$. In fact, $C$ is the product $A \cdot B$ of two triangular matrices with $1^{\prime} s$ along their diagonals, with $A=\left(a_{i, j}\right)$ lower triangular, and $B=\left(b_{i j}\right)$ upper triangular:

$$
a_{i j}=\left(\begin{array}{c}
2 i-j-1 \\
j-1
\end{array}\right), i \geq j ; \quad b_{i j}=\frac{j}{i}\left(\begin{array}{c}
i \\
j-i
\end{array}\right), i \leq j .
$$

The proof that $A \cdot B=C$ follows from the following identity among binomial coefficients, whose proof was showed to me by R. Narasimhan.

Lemma. For positive integers $m$ and $n$,

$$
\sum_{k} \frac{1}{m+n-k}\left(\begin{array}{c}
m+n-k \\
n
\end{array}\right)\left(\begin{array}{c}
n \\
2 k-n
\end{array}\right)=\frac{1}{m+n}\left(\begin{array}{c}
m+n \\
n
\end{array}\right) .
$$

The sum is over all integers $k$, but there are nonzero contributions only for $m / 2 \leq k \leq \min (m,(m+n) / 2)$.

Proof. Let $f(x)=\frac{1}{n}\left(1-x^{2}\right)^{-n}=\sum_{k} \frac{1}{n+k}\left(\begin{array}{c}n+k \\ n\end{array}\right) x^{2 k}$, and $g(x)=$ $\frac{1}{2}\left[(1+x)^{n}+(1-x)^{n}\right]=\sum_{k}\left(\begin{array}{c}n \\ 2 k\end{array}\right) x^{2 k}$. If $m$ is even, the coefficient of $x^{m}$ in $f \cdot g$ is the left side of the equation in the lemma. Since

$$
f \cdot g=\frac{1}{2 n}\left[(1-x)^{-n}+(1-x)^{-n}\right]=\frac{1}{n} \sum_{k}\left(\begin{array}{c}
-n \\
2 k
\end{array}\right) x^{2 k},
$$

the coefficient of $x^{m}$ in $f \cdot g$ is $\frac{1}{n}\left(\begin{array}{c}-n \\ m\end{array}\right)=\frac{1}{m+n}\left(\begin{array}{c}m+n \\ n\end{array}\right)$, as required.

If $\mathrm{m}$ is odd, the argument is similar, replacing $g(x)$ by

$$
\left[(1+x)^{n}-(1-x)^{n}\right] / 2 x=\sum_{k}\left(\begin{array}{c}
n \\
2 k+1
\end{array}\right) x^{2 k}
$$

and calculating the coefficient of $x^{m-1}$ in $f \cdot g$.

\section{References}

[1] I. N. Bernstein, I. M. Gelfand \& S. I. Gelfand, Schubert cells and cohomology of the spaces $G / P$, Russian Math. Surveys 28:3 (1973) 1-26.

[2] A. Bertram, An existence theorem for Prym special divisors, Invent. math. 90 (1987) 669-671.

[3] S. Billey \& M. Hairnan, Schubert polynomials for the classical groups, J. Amer. Math. Soc. 8 (1995) 443-482.

[4] S. Billey, W. Jockusch \& R. P. Stanley, Some combinatorial properties of Schubert polynomials, J. Algebraic Combinatorics 2 (1993) 345-374. 
[5] C. De Concini \& P. Pragacz, On the class of Brill-Noether loci for Pryrn varieties, Preprint, 1994.

[6] M. Demazure, Désingularization des variétés de Schubert généralisées, Ann. Scuola Norm. Sup. Pisa Cl. Sci. (4) 7 (1974) 53-88.

[7] D. Edidin \& W. Graham, Characteristic classes and quadric bundles, Duke Math. J. 78 (1995) 277-299.

[8] W. Fulton, Intersection Theory, Springer-Verlag, 1984.

[9] _ Flags, Schubert polynomials, degeneracy loci, and determinantal formulas, Duke Math. J. 65 (1991) 381-420.

[10] __ Schubert varieties in flag bundles for the classical groups, to appear in Proceedings of Conference in Honor of Hirzebruch's 65th Birthday, Bar Ilan, 1993 Israel Mathematical Conference Proceedings 9 (1995), Amer. Math. Soc.

[11] W. Fulton \& R. Lazarsfeld, On the connectedness of degeneracy loci and special divisors, Acta Mathematica 146 (1981) 271-283.

[12] - The numerical positivity of ample vector bundles, Annals of Math. 118 (1983) 35-60.

[13] G. Z. Giambelli, Sulle varietà rappresentate coll'annullare determinanti minori contenuti in un determinante simmetrico ad emisimmetrico generico di forme, Atti della R. Acc. delle Scienze di Torino 41 (1905/06) 102125.

[14] J. Harris \& L. W. Tu, On symmetric and skew-symmetric determinantal varieties, Topology 23 (1984) 71-84.

[15] T. Jòzefiak, A. Lascoux \& P. Pragacz, Classes of determinantal varieties associated with symmetric and skew-symmetric matrices, Math. USSR Izvestija 18 (1982) 575-586.

[16] V. Lakshmibai \& C. S. Seshadri, Standard Monomial Theory, pp. 279-322 in Proc. Hyderabad Conf. on Algebraic Groups, 1989 (S. Rarnanan, ed.), Manoj Prakashan, Madras, 1991.

[17] I. G. Macdonald, Notes on Schubert Polynomials, Publications du LACIM, Universitè du Quèbec à Montrèal, 1991.

[18] D. Mumford, Theta characteristics of an algebraic curve, Ann. scient. Èc. Norm. Sup. 4 (1971) 181-192.

[19] P. Pragacz, Enumerative geometry of degeneracy loci, Ann. Sci. Ecole Norm. Sup. 21 (1988) 413-454.

[20] _ Cycles of isotropic subspaces and formulas for symmetric degeneracy loci, pp. 189-199 in Topics in Algebra, Banach Center Publications, Vol 26, Part 2, 1990.

[21] _ Algebro-geometric applications of Schur S-and Q-polynomials, Springer Lecture Notes 1478 (1991) 130-191.

[22] P. Pragacz \& J. Ratajski, Pieri type formula for isotropic Grassrnannians; the operator approach, Manuscripta Math. 79 (1993) 127-151.

[23] _ An intersection formula for Grassmannians of non maximal isotropic subspaces, Preprint, 1992.

[24] 
polynomials approach, Preprint, 1994.

University of Chicago, Chicago 